\title{
Switching between self-renewal and lineage commitment of human induced pluripotent stem cells via cell-substrate and cell-cell interactions on a dendrimer-immobilized surface
}

Mee-Hae Kim, Masahiro Kino-oka*

Department of Biotechnology, Graduate School of Engineering, Osaka University, 2-1 Yamadaoka, Suita, Osaka 565-0871, Japan

*Corresponding author: Masahiro Kino-oka, Ph.D.

Tel.: +81-(0)6-6879-7444; fax: +81-(0)6-6879-4246

E-mail address: kino-oka@bio.eng.osaka-u.ac.jp 


\section{ABSTRACT}

Understanding mechanisms that govern cell fate determination of human induced pluripotent stem cells (hiPSCs) could assist in maintenance of the undifferentiated state during cell expansion. We used polyamidoamine dendrimer surfaces with first-generation (G1), third-generation (G3) and fifth-generation (G5) of dendron structure in cultures of hiPSCs with SNL feeder cells. Cells on the G1 surface formed tightly packed colony with close cell-cell contacts during division and migration; those on the G3 surface exhibited loose or dispersed colony pattern by enhanced migration. On the G5 surface, formation of aggregated colony with ring-like structures occurred spontaneously. We found that the substrate-adsorbed fibronectin and feeder cell-secreted fibronectin appeared elevated levels with the varied generation numbers of dendrimer surfaces. This subsequently resulted in cell migration and in activation of paxillin of hiPSCs. Location-dependent expression of Rac1 induced rearrangement of E-cadherin-mediated cell-cell interactions on dendrimer surfaces, and was associated with alterations in the cell and colony morphology, and migratory behavior. Furthermore, Caspase-3 occurred in apoptotic cells on dendrimer surfaces, concomitant with the loss of E-cadherin-mediated cell-cell interactions. Cells on the G1 surface were maintained in an undifferentiated state, while those on the G5 surface exhibited the early commitment to differentiation toward endodermal fates. We conclude that morphological changes associated with altered migration on the dendrimer surfaces were responsible for the coordinated regulation of balance between cell-cell and cell-substrate interactions associated with migration, thereby switching their transition from self-renewal state to early endoderm differentiation in hiPSCs.

Keywords: Human induced pluripotent stem cells, Dendrimer surface, Fibronectin adsorption, Cell migration, Cell-cell interaction, Cell-substrate interaction, Cell fate decision 


\section{Introduction}

Induced pluripotent stem cells (iPSCs) were originally generated from mouse and human fibroblasts via the retroviral introduction of Oct3/4, Sox2, c-Myc and Klf4 [1-3]. These iPSCs are similar to embryonic stem cells (ESCs) in terms of their morphology, gene expression, pluripotency and self-renewal [4]. They represent an important advance in stem cell research, as they allow researchers to obtain pluripotent stem cells, which are important in research and potentially have therapeutic uses, without the controversial use of embryos. Therefore, iPSCs have great potential for a wide range of applications in regenerative medicine, and as tools for human disease modeling and drug discovery.

Cell expansion and directed differentiation of iPSCs remains a challenging process because of spontaneous differentiation, which is accompanied by a loss in self-renewal and pluripotent qualities during maintenance culture [5-9]. To regulate the fate decision of hiPSCs, many researchers have attempted to design microenvironments that mimic the stem cell niche, thereby driving cells down their preferred self-renewal and differentiation pathway [7-11]. Many researches have shown that stem cells reside in a complex microenvironment, and their fate is determined by several factors, such as soluble and physical signals from extracellular matrix and neighboring cells. Additionally, a fundamental understanding of the cell-cell and cell-substrate interactions is required for the design the artificial environments $[11,13,15]$. There are few reports focusing on key regulatory mechanism that control their transition from a pluripotent to differentiated state in maintenance cultures.

Culture surface topography has an effect on morphology and gene expression. It is surprising that relatively little work has been done to delineate the effects of surface topography on the processes involved in the synthesis and secretion of proteins by cells cultured on surfaces with differing topographies $[8,16]$. One protein of particular interest in determining the relationship between cells and their substratum is fibronectin [16]. Fibronectin is one of the major molecules mediating cell attachment, and is likely affected by substrata, which alter the distribution of 
cytoskeletal elements [16]. A number of reports have shown that cell adhesion molecule-mediated interactions among iPSCs, their neighboring instructive/support cells, and extracellular matrix significantly contribute to the pluripotent state of iPSCs [9-15]. Cell-cell and cell-substrate interactions of iPSCs lead to changes in cell and colony morphology, which potentially activates signalling pathways involved in either maintaining the undifferentiated state or committing to a lineage. The development of culture substrate has been reported to regulate fate of iPSCs [9]. Although there are reports of the development of defined synthetic substrates, such as small peptides, to allow for the regulation of iPSC fate, considerable improvements and refinements of these substrates are required.

The use of a dendrimer surface has been proposed as a method of regulating the morphology and function of cells [17-20]. Several concepts of morphological change on dendrimer surfaces have been proposed, including ligand display control over cells caused by the binding balance between integrin on the cytoplasm and adhesion proteins on surface [17]. Changes in cell morphology are particularly dependent on the generation number of the dendrimer surface $[17,18]$. Alterations of the generation number of the dendrimer possibly promote the cellular morphological changes through active migration, and can be accompanied by dynamic cytoskeletal formation in the cells on the culture surface [18].

In this study, we examined the morphological and functional responses of human iPSCs (hiPSCs) cultured on the dendrimer-immobilized surface with feeder cells. Based on examining morphological behaviors, fundamental mechanisms of cell-cell and cell-substrate interactions are discussed in aspects of survival, self-renewal and early lineage commitment of hiPSCs.

\section{Materials and Methods}

\subsection{Cells and culture conditions}

hiPSCs were provided from Japanese Collection of Research Bioresources (JCRB) Cell Bank (clone Tic, JCRB Number: JCRB1331). Routine subcultures of hiPSCs were conducted in a culture 
dish (surface area; $55-\mathrm{cm}^{2}$, Corning Costar, Cambridge, MA, USA) using commercially available medium (ReproStem, ReproCELL Inc., Tokyo, Japan) containing $5 \mathrm{ng} / \mathrm{ml}$ basic fibroblast growth factor on the $0.1 \%$ gelatin-coated surfaces with feeder layer of mitomycin C-treated SNL76/7 cells (European Collection of Cell Cultures, Salisbury, UK) at $37^{\circ} \mathrm{C}$ under a $5 \% \mathrm{CO}_{2}$ atmosphere. Culture medium was changed daily. For routine passages of hiPSCs, feeder cells were removed after a 1-min incubation with CTK solution as described elsewhere [2]. The hiPSC colonies in the undifferentiated state were carefully collected using a cell scraper (Sumitomo Bakelite Co. Ltd., Osaka, Japan). Suspension of collected undifferentiated colonies was gently pipetted for dispersal into small aggregates, and then dispensed into a fresh culture vessel containing feeder cells.

For the preparation of feeder layer, mitomycin C-treated SNL76/7 cells were cultured in advance for $24 \mathrm{~h}$ in Dulbecco's modified Eagle's medium (DMEM, Sigma-Aldrich, MO, USA) supplemented with 7\% fetal bovine serum (FBS, Invitrogen, Grand Island, NY, USA) with antibiotics $\left(100 \mathrm{U} / \mathrm{cm}^{3}\right.$ penicillin $\mathrm{G}, 0.1 \mathrm{mg} / \mathrm{cm}^{3}$ streptomycin and $0.25 \mathrm{mg} / \mathrm{cm}^{3}$ amphotericin $\mathrm{B}$; all obtained from Invitrogen). The seeding density was fixed at a viable cell concentration of $2.5 \times 10^{4}$ cells $/ \mathrm{cm}^{2}$.

\subsection{Culture on dendrimer surface}

The dendrimer surface was prepared using the conventional tissue culture polystyrene (PS) surface of a square 8-well plate (surface area; $10.5 \mathrm{~cm}^{2}$, Nunc, Roskilde, Denmark), as described previous report [17]. Briefly, first-generation (G1), third-generation (G3), and fifth-generation (G5) surfaces were created, under sterile conditions, by changing the generation number of synthesized dendrimers over four reactions. Hydroxyl groups were displayed on the PS surface by pouring potassium tert-butoxide into the wells. Then, aqueous glutaraldehyde was introduced into the wells, with the wells were then treated with a tris(2-aminoethyl) amine solution to produce a dendron structure. The wells were rinsed with sterile water. To display glucose as a terminal ligand, D-glucose was applied.

For the hiPSC cultivation, SNL76/7 feeder cells were cultured on the prepared dendrimer 
surfaces. The hiPSCs were plated on dendrimer surface with SNL feeder cells under same conditions as those described above. Time-lapse observation for the morphology of cells and colonies was conducted by obtaining images every $10 \mathrm{~min}$ at several positions using a custom-made observation tool [21].

\subsection{Immunofluorescence staining}

The procedure used for immunofluorescence staining was similar to that described previously [18]. Briefly, hiPSCs were fixed with 3.7\% paraformaldehyde (Wako Pure Chemical Industries, Osaka, Japan) for $10 \mathrm{~min}$ at room temperature and rinsed with PBS, followed by soaking in PBS with $0.25 \%$ Triton X-100 for 4 min. After masking of non-specific proteins by incubation in Block Ace (Dainippon Sumitomo Pharma Co., Ltd., Osaka, Japan) for $1 \mathrm{~h}$ at ambient temperature, the cells were treated with a primary antibody at $4{ }^{\circ} \mathrm{C}$ overnight. Specifically, the cells were incubated with anti-Paxillin (clone 15D2, Zymed/Invitrogen), anti-Rac1 (Santa Cruz Biotechnology, Santa Cruz, CA), anti-E-cadherin (Takara Biomedicals, Shiga, Japan), anti-cleaved Caspase-3 (Cell Signaling Technology Inc. Beverly, MA, USA), and anti-Oct3/4 (Santa Cruz Biotechnology) primary antibodies that were adequately diluted in PBS containing 10\% Block Ace. Cells were washed with Tris-buffered saline followed by immunolabeling with Alexa Fluor 488-conjugated goat anti-rabbit or Alexa Fluor 594-conjugated goat anti-mouse IgG (Molecular Probes) for 1h. Cell nucleus were stained with 4',6-diamidino-2-phenylindole (DAPI). Images were obtained using an image analyzer

(In CELL Analyzer 2000; GE Healthcare, Buckinghamshire, UK) through 10× objective lens and confocal laser scanning microscope (Model FV-1000; Olympus, Tokyo) through $60 \times$ objective lens.

\subsection{Protein extraction and western blot analysis}

Protein extraction and western blot analysis were conducted using similar method as described previously [16]. Total cellular protein was extracted from cells using CelLytic MT mammalian cell lysis/extraction reagent (Sigma-Aldrich). Total proteins in conditioned media were precipitated with trichloroacetic acid at $4^{\circ} \mathrm{C}$ for $30 \mathrm{~min}$. Equal quantities of protein lysates were separated by sodium dodecyl sulfate polyacrylamide gel electrophoresis and transferred to nitrocellulose membranes 
using standard methods. Blots were blocked in 5\% nonfat dry milk in Tris-buffered saline (TBS), $\mathrm{pH} 7.4$ before incubation with in primary antibodies in 5\% milk/TBS. Blots were rinsed three times and washed four times $5 \mathrm{~min}$ in TBS $+0.1 \%$ Tween 20 (TBST). Blots were rinsed three times and were incubated with horseradish peroxidase-conjugated secondary antibodies in 5\% milk/TBS, and then rinsed and washed in TBST. Using methods describe previously [19], proteins were transferred to membranes, incubated with primary antibodies, and then visualized with an Enhanced Chemiluminescence detection system (Amersham Bioscience, Piscataway, NJ, USA). Fibronectin adsorption was investigated with a bovine-specific anti-fibronectin antibody (Chemicon/Millipore, Billerica, MA, USA) which permits the selective detection of fibronectin derived from FBS. Blots were reprobed with anti-glyceraldehyde-3-phosphate dehydrogenase (GAPDH) antibody (Sigma-Aldrich) as a loading control.

\section{6. $R$ NA isolation and quantitative polymerase chain reaction (qPCR) assays}

RNA isolation, cDNA synthesis, and PCR analysis were conducted as described previously [19]. Total RNA were isolated using an RNeasy Mini Kit (Qiagen, Hilden, Germany) and TRIzol (Invitrogen). One $\mu \mathrm{g}$ of total RNA was then reverse transcribed using SuperScript II Reverse Transcriptase kit (Invitrogen). The qPCR assays were conducted using SYBR Premix Ex Taq (Takara Shuzo, Shiga, Japan), and contained $1 \mu \mathrm{l}$ of cDNA per $25 \mu \mathrm{l}$ of reaction mixture. Assays were carried out on a 7300 Real-Time PCR System (Applied Biosystems, Foster City, CA, USA). The relative quantity of the target transcript was estimated by a standard curve, and the data were standardized based on the GAPDH expression. All PCR products were checked by melting curve analysis to exclude the possibility of multiple products or incorrect product size. The sequences of oligonucleotide primers we used are listed in Supplementary table S1.

\section{Results}

\subsection{Adsorption and secretion of fibronectin matrix on dendrimer surfaces}

To examine the initial fibronectin adsorption onto dendrimer surface, the cultures of feeder 
cells were performed on G1, G3, and G5 surfaces in DMEM containing 10\% FBS for $24 \mathrm{~h}$. Since a large fraction of extracellular molecules may bind to the cell surface and be assembled into extracellular matrix shortly after being secreted into the medium [16], the fibronectin of conditioned media (media fibronectin) and cell layer extract (matrix fibronectin) was detected by western blotting. As shown in Fig. 1A, the matrix fibronectin was detected more high level in the cells on Fig. 1 G5 surface, compared with those in cultures on the G1 and G3 surfaces. Fibronectin in the media predominantly appeared in cultures where cells growing on G1 and G3 surfaces, which in turn exhibited low levels of matrix fibronectin.

We also tested the fibronectin secreted from feeder cells in culture of hiPSCs on the G1 and gelatin-coated surfaces by real-time PCR assay at 5 days. As shown in Fig. 1B, fibronectin were expressed at higher levels in the feeder cells on G5 surface, compared with those in cultures on the G1 and G3 surfaces. These findings indicated that dendrimer surface enhanced the initial adsorption of fibronectin and fibronectin secretion from feeder cells.

\subsection{Formation of organization of cytoskeletal proteins of hiPSCs}

To understand the dynamic behavior of hiPSCs on dendrimer surfaces, time-lapse observation of hiPSCs on the G1, G3, and G5 surfaces with feeder cells was conducted. All the observed cells inside colonies were found to possess migratory potentials. Cells on the G1 surface grew in rounded, tightly packed colonies with defined borders at the periphery of the colony through division and migration (Movie S1 and Fig. 2C). Cells on the G3 surface grew in scattered colony associated with Fig. 2 active migration (Movie S2, and Fig. 2F), with cells becoming partially apoptotic. Cells on the G5 surface exhibited characteristic concentric ring-like patterns with aggregation through division as well as coalescence with one another (Movie S3, and Fig. 2I).

Subsequently, the results of immunostaining of paxillin and F-actin showed notable differences in their expression levels that were dependent on location. Expressions of paxillin and F-actin in cells cultured on G1, G3, and G5 surfaces with feeder cells for 5 days were observed. As shown in Fig. 3, actin stress fibers on the G1 surface had a linear morphology along the boundaries Fig. 3 
between adjacent cells, with a nebulous distribution of paxillin expression observed for areas of cell-cell contacts at the basal side (Fig. 3C). In the stretched cells on the G3 surface, distinct and straight stress fibers were distributed in cells inside colony including the leading edge with lamellipodia, and especially at the ends of the stress fibers with intensive scattered staining in a longitudinal direction (Fig. 3F). For the aggregated cells on the G5 surface, the formation of contractile stress fibers became pronounced, with paxillin indiscriminately distributed throughout the aggregate cells (Fig. 3I).

\subsection{Activities and localization of E-cadherin and Rac1inside hiPSC colony}

To investigate whether the cytoskeletal formation derived on the dendrimer surfaces was associated with the cell migration, we performed immunostainings to determine the expression of a cell-cell contact-associated protein, E-cadherin and a member of the Rho family GTPases, Rac1 for 5 days. As shown in Fig. 4, E-cadherin was expressed in all cells inside colony on G1 surface. Cells Fig. 4 expressing E-cadherin exhibited a cuboidal morphology, and formed cobblestone colonies, and were observed as a continuous line at the boundaries between neighboring cells (Fig. 4C and 4D). Notable differences were seen for the distribution of E-cadherin and Rac1 expression, which were dependent on location. A comparison of central and peripheral regions for a single colony revealed that the Rac1 were highly concentrated at the periphery (Fig. 4A and 4B). In cells on the G3 surface, Rac1 was strongly expressed in almost all the cells, and appeared to be distributed throughout the cytoplasm of cells lacking E-cadherin expression (Fig. 4I and 4J). For cells on the G5 surface, E-cadherin and Rac1 was distributed throughout aggregated cells (Fig. 4N and 4M). These findings indicate that the alteration of cell migration inside hiPSC colonies is associated with the rearrangement of E-cadherin-mediated cell-cell contacts inside colony.

\subsection{Apoptosis-induced cleaved Caspase-3 associated with E-cadherin inside hiPSC colony}

To understand the mechanism of the effect of cell-cell contacts on the apoptosis, immunostaining for E-cadherin, and an apoptotic cell death-associated protein, cleaved Caspase-3, was conducted in single colonies cultured on G1, G3 and G5 surfaces for 5 days. As shown in Fig. 5, Fig. 5 
the cleaved Caspase-3 was not detected in the cells on the G1 surface, although they were prominently seen in cells on the G3 and G5 surfaces. Cleaved Caspase-3 was expressed in the scattered cells and aggregated cells with lower levels of E-cadherin expression at border between adjacent cells (Fig. 5I and 5J). These results indicate that the rearrangement of E-cadherin-mediated cell-cell contacts on dendrimer surface can be lead to alternation of their transition from survival to death inside hiPSC colony.

\subsection{Self-renewal and early lineage commitment inside hiPSC colony}

To confirm the state of undifferentiated hiPSCs cultured on the dendrimer surfaces for 5 days, immunostaining for undifferentiated state marker Oct3/4 was performed. As shown in Fig. 6A, all Fig. 6 of the cells inside hiPSC colony on the G1 surface were Oct3/4 positive. However, Oct3/4 was only partially detected in the scattered cells from these colonies and in the uppermost layer of aggregated cells on G3 and G5 surfaces (Fig. 6A2 and Fig. 6A3).

Next, we carried out the gene expressions of pluripotency and early lineage commitment of the hiPSCs cultured on dendrimer surfaces for 5 days by using qPCR assays. Growth on gelatin-coated surface was used as a reference condition for comparing the growth and maintenance of hiPSCs on the dendrimer surfaces. As shown in Fig.6B, the expressions of the pluripotency markers Oct3/4, Nanog, and Sox2 in cells cultured on G1 surface were similar to those on gelatin-coated surface. Additionally, we found that markers for all three germ layers were not detected in cells on the G1 surface. However, expression levels of early endodermal marker GATA4 in cells cultured on the G5 surface was apparent, however, this was not seen in cells grown on the G1 and G3 surface. Therefore, it is considered that dendrimer surface exhibited the role of regulators of hiPSC fate decisions from self-renewal state to early endoderm lineages.

\section{Discussion}

\subsection{Fibronectin matrix on dendrimer surface regulates adhesion and morphology of hiPSCs}

Interaction of cells via integrin with culture substrate leads to changes in the abundance or 
composition of adhesion complexes. This potentially activates signalling pathways involved in either maintaining undifferentiated state, or committing stem cells down a certain lineage [7-9]. The initial cell-material interaction is a complex multi-step process consisting of early events, such as adsorption of proteins, followed by cell adhesion and spreading, and late events, related to cell growth, differentiation, matrix deposition, and cell functioning [16]. Integrin-mediated adhesions regulates the focal adhesion-associated signaling molecule, paxillin. The tyrosine phosphorylation of paxillin is known to be important for regulating signals from Rho family GTPases to the actin cytoskeleton [25-27]. Paxillin was observed following integrin-dependent cell adhesion to matrics protein such as fibronectin onto culture surface [26]. The binding $\alpha 5 \beta 1$ integrin to specific fibronectin domains specifies differentiation into mesodermal and ectodermal lineages [25]. Paxillin positively regulates Rac1 activity and negatively regulating RhoA. Additionally, Rac1 up-regulation promotes cadherin-mediated cell-cell adhesion, indicating that E-cadherin-mediated cell-cell adhesion has a close correlation with directing cell fate via connections between cadherin complexes [28-30]. These previous studies have revealed that paxillin-mediated up-regulation of Rac1 at the culture surface functions in the recruitment of diverse cytoskeletal and signaling proteins into a complex, presumably to coordinate the transmission of downstream signals that can determine hiPSC fates.

Polyamidoamine dendrimer-immobilized surface have been shown to cause morphological changes, and alter the function of cells [17-20]. Morphological changes are accompanied by actin cytoskeleton organization and transduction of intercellular signaling in cells on the culture surface. In this study, we found that changes in cell morphology and colony formation of hiPSCs occurred through altered migration on the modified surface, which varied with generation number. When hiPSCs were cultured on the G1 surface, cells grew in rounded, tightly packed colony and maintain defined borders at the periphery of colony with close cell-cell contact during division and migration (Movie S1 and Fig. 2C). A significant increase in migration of hiPSCs was seen on the G3 surface; cells were dispersed throughout colonies and less cell-cell interactions were seen, resulting in 
apoptosis and loss of pluripotency (Movie S2 and Fig. 2F). When hiPSCs were cultured on G5 surface, they formed self-aggregated colonies with a concentric ring-like structure (Movie S3 and Fig. 2I). To determine the effect of dendrimer surface on morphology of cells and colonies, we investigated the amount of fibronectin adsorbed onto the dendrimer surfaces for 5 days. We found that the substrate-adsorbed fibronectin and cell secreted fibronectin on dendrimer surfaces appeared elevated levels with the varied generation numbers (Fig. 1). This subsequently resulted in cell migration and in activation of paxillin and further Rac1 of hiPSCs (Movie S3, Fig. 3 and Fig. 4). These results suggest that a dendrimer surface with a cationic charge is responsible for fibronectin adsorbed onto the dendrimer surfaces and that fibronectin is secreted from feeder cells. Immunostainings with Rac1 and E-cadherin revealed that the changes in cell migration on the dendrimer surfaces were accompanied by location-dependent expressions of Rac1, and were concomitant with the regulation of E-cadherin-mediated cell-cell interactions (Fig. 4). It is most likely that morphology and cytoskeletal formation was ultimately determined by a moderate balance between cell-cell and cell-substrate interactions, via the rearrangement of integrin-mediated interactions at the culture surface. These studies support the hypothesis that extracellular signals can be locally integrated by the endogenous Rho family GTPases to coordinate the balance between cell-cell and cell-substrate interactions that are necessary for directing fates of stem cells.

\subsection{Alternation of balance between cell-cell and cell-substrate interactions by migration controls} hiPSC fates between self-renewal and lineage commitment

Colony formation is an important event influencing the fate of hiPSCs. Mechanisms of colony formation in vitro are needed to be elucidated to understand how and why these cells choose between self-renewal and lineage commitment. Integrin- and cadherin-mediated interactions have been considered essential for colony formation of hiPSCs in a pluripotent state [28]. Cadherin-mediated cell-cell interaction is associated with Rho family GTPase activities and is related to cytoskeleton formation, offering a signaling pathway for the generation of E-cadherin-mediated cell-cell interactions that underlie self-renewal and maintenance of 
undifferentiated hiPSCs. As an example, up-regulation of E-cadherin expression enhances the self-renewal capacity of human ESCs through up-regulation of Nanog and Oct3/4 genes via nuclear translocation [11-13]. Conversely, the down-regulation of E-cadherin stimulates Caspase-3, pro-apoptotic gene, and suppresses $B c l-X L$, apoptotic inhibitory gene, resulting in an increase in cell death $[31,32]$.

In this study, we have described a possible mechanism by which niche components influence cell fate decisions during hiPSC culture. We have also successfully incorporated these concepts into the design of biomimetic microenvironments (Fig. 7). Ultimately, an appropriate balance between Fig. 7 interactions of cell-cell and cell-substrate forms the undifferentiated colonies. With an increase in the cell-substrate interactions, cells exhibit very loose or dispersed colony pattern because of enhanced migration, leading to apoptosis. A decrease in cell-substrate interactions tilts the balance to a state more favorable for aggregate formation, resulting in differentiation of hiPSCs to early endodermal fates. Immunostainings with E-cadherin and Caspase-3 revealed that the occurrences of apoptotic cells derived on dendrimer surface were accompanied by location-dependent expressions of Caspase-3, and were concomitant with the loss of E-cadherin-mediated cell-cell interactions (Fig. 5). Immunostaining and gene expression of pluripotency and early differentiation markers demonstrated that the cells on G1 surface were maintained in an undifferentiated state (Fig. 6). Cells cultured on the G5 surface exhibited the differentiation of early commitment to endodermal fates. The fate of hiPSCs with respect to survival, self-renewal, or early lineage commitment can be explained in terms of the balance between cell-cell and cell-substrate interactions, which can be altered cell migration on dendrimer surfaces. We propose that the coordinated regulation of balance between cell-substrate and cell-cell interactions through altered migration is a key regulatory mechanism in maintenance of its survival, self-renewal, and lineage commitment of hiPSCs. Taken together, our findings suggest that dendrimer surface might have a role as model substrate for guiding proper hiPSC fates in an ex vivo stem cell culture system. 


\section{Conclusions}

The present study suggests that altered migrations can lead hiPSCs to alter their differentiation fate when cultured on dendrimer surfaces in the presence of feeder cells. We found that altering the generation number of dendrimer surface promoted morphological change in hiPSCs while actively migrating. We showed that altering the balance between cell-cell and cell-substrate interactions through migration played an important role in controlling the fate of cells. The endogenous Rac 1 and E-cadherin signaling pathways are critical in the regulation of cellular self-renewal, while commitment down a particular differentiation pathway was dependent upon a modulating network of transcription factors. Dendrimer surface offers a model of designing a substrate, based on interactions between the microenvironment and cells, which could be useful for obtaining cell preparations enriched in hiPSCs in an undifferentiated state, or committed towards an early endodermal fate.

\section{Acknowledgements}

We thank T. Iga for help with experiments and analyses. This work was partially supported by the Japan Science for the Promotion of Science (JSPS), Grants-in-Aid for Young Scientists (B) (Nos. 23760749 and 25820401), and Grants-in-Aid for Scientific Research (B) (No.21360402) from the Ministry of Education, Culture, Sports, Science and Technology (MEXT) of Japan. Our work was also supported by the Strategic Promotion of Innovative Research and Development (S-Innovation) Program of the JST, and the JSPS through the "Funding Program for World-Leading Innovative R\&D on Science and Technology (FIRST Program)" initiated by the Council for Science and Technology Policy (CSTP). 


\section{Figure 1}

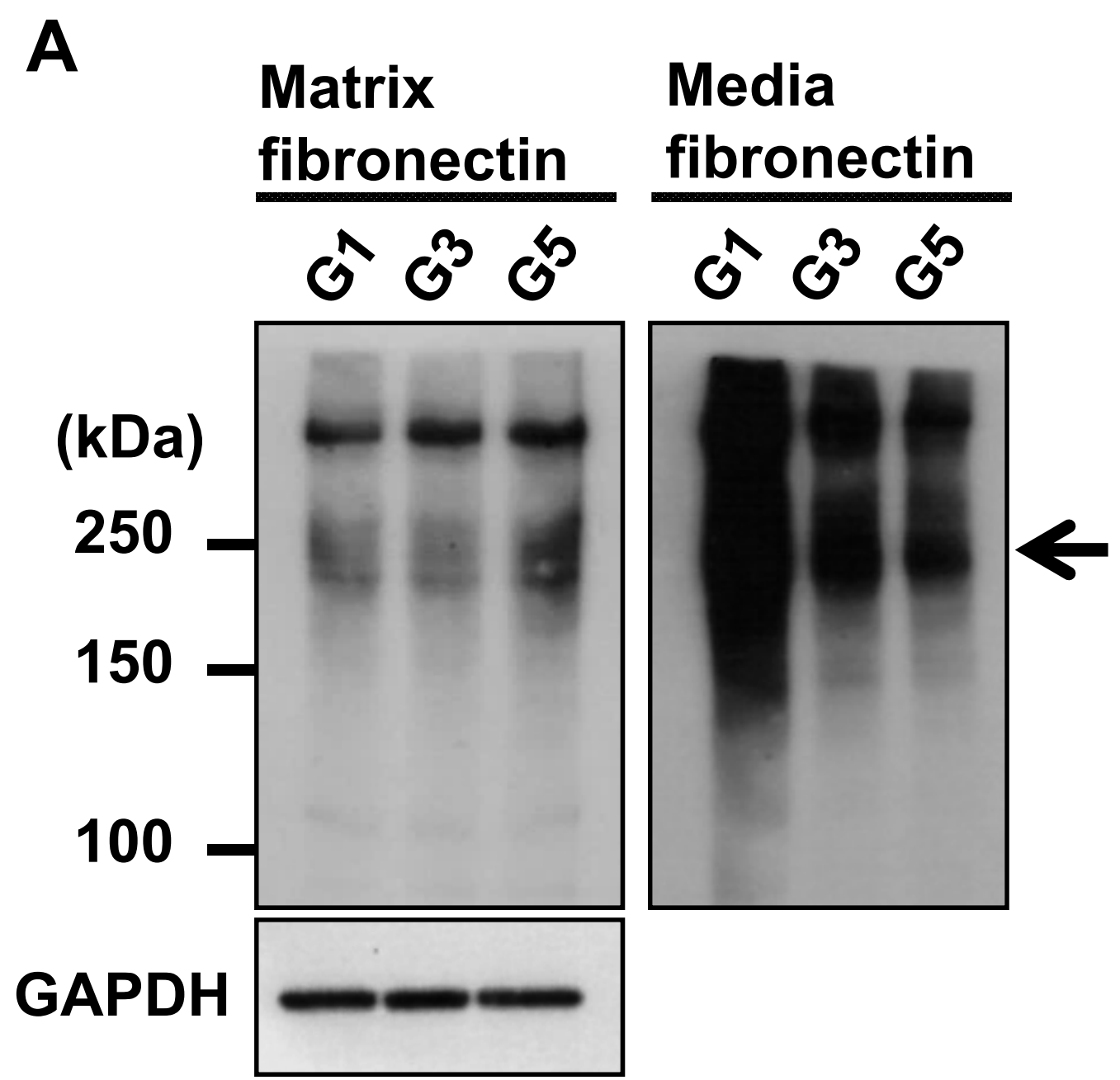

B

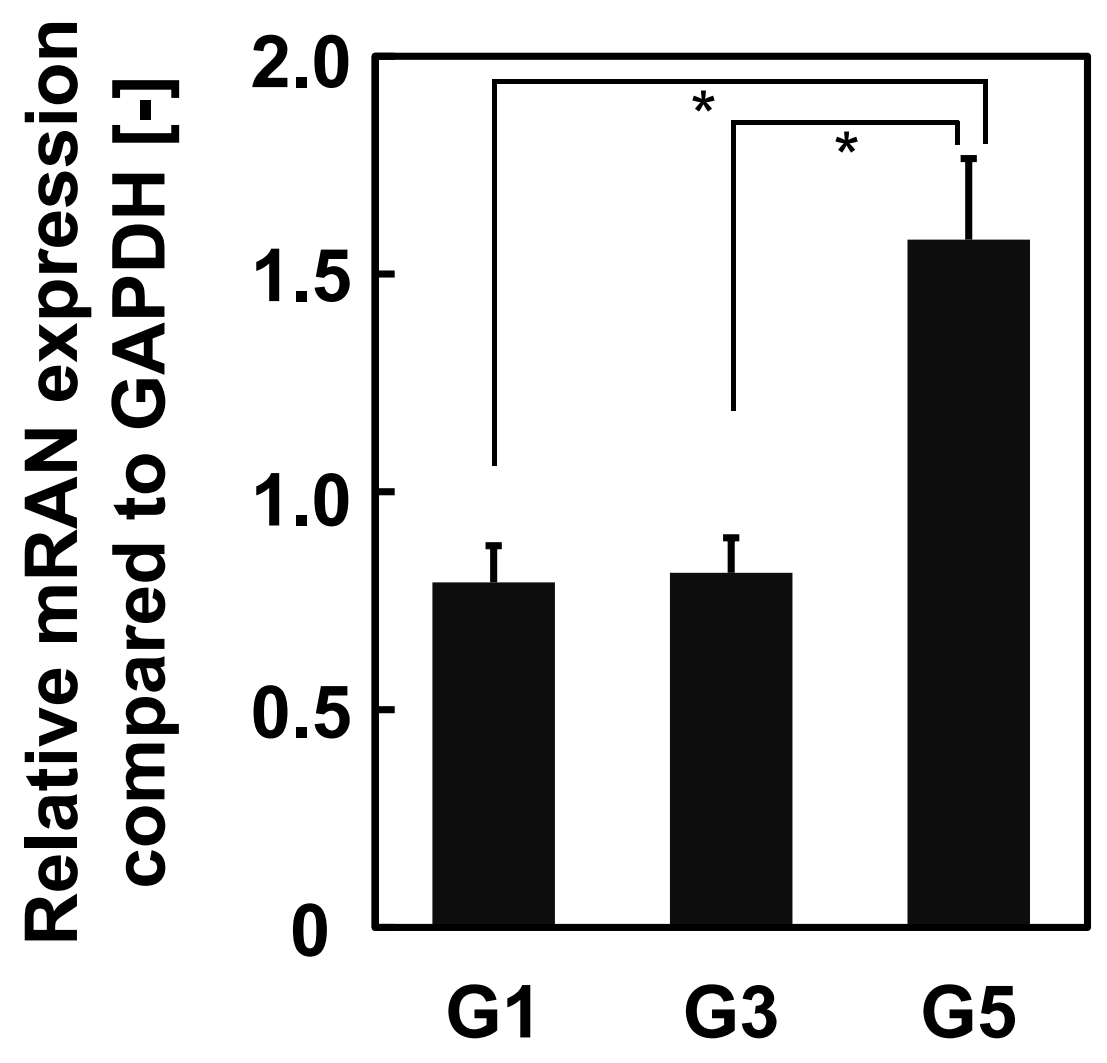




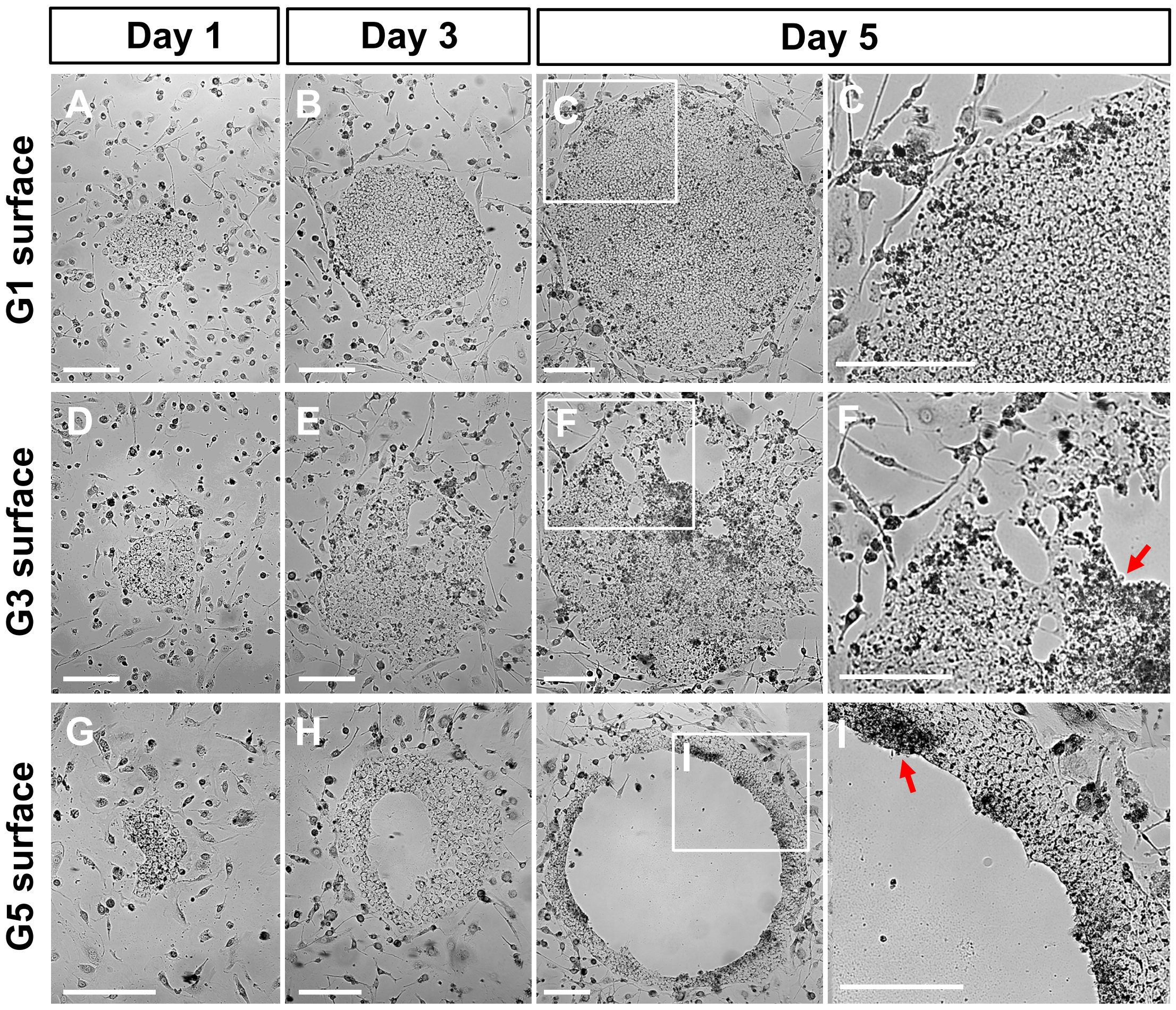




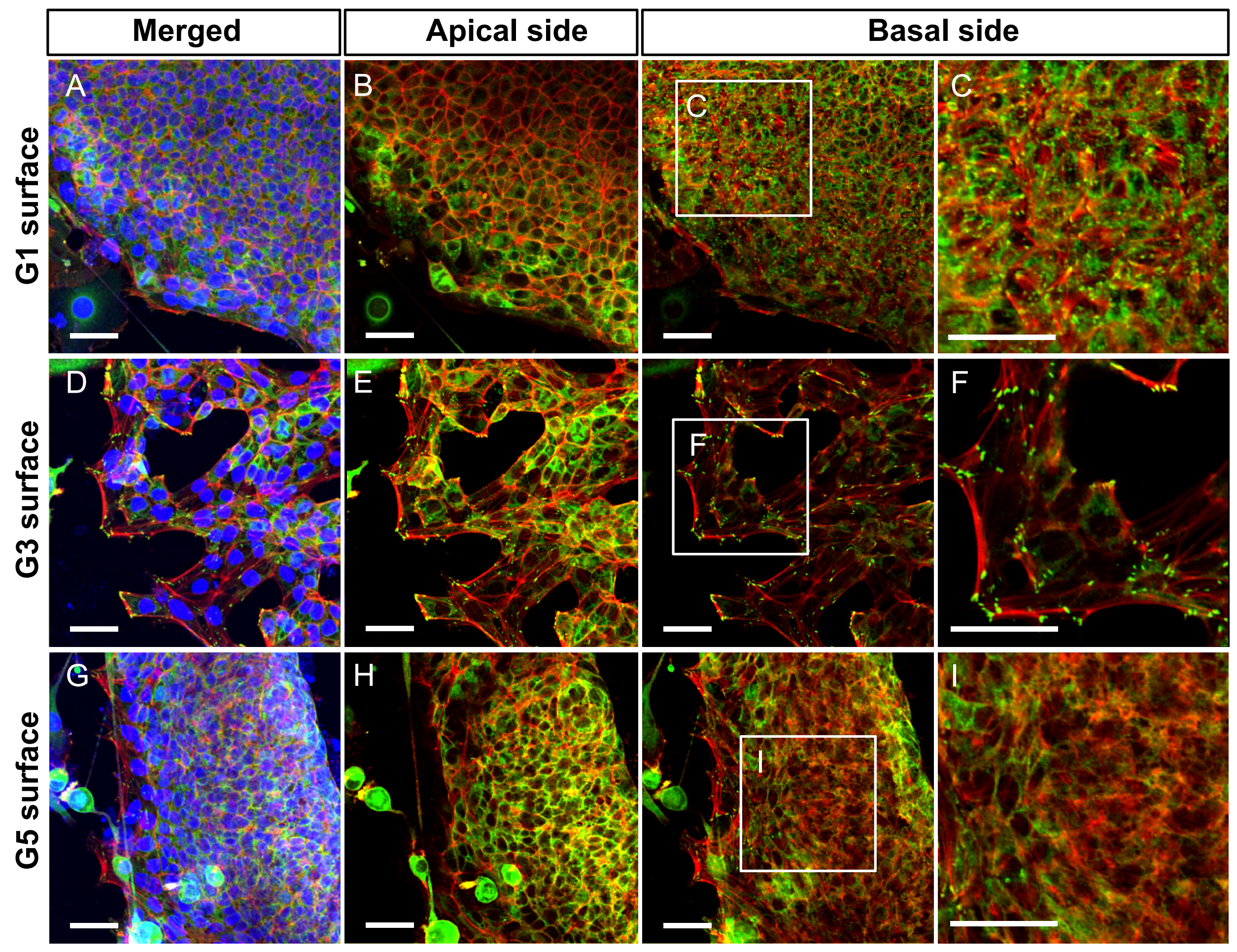




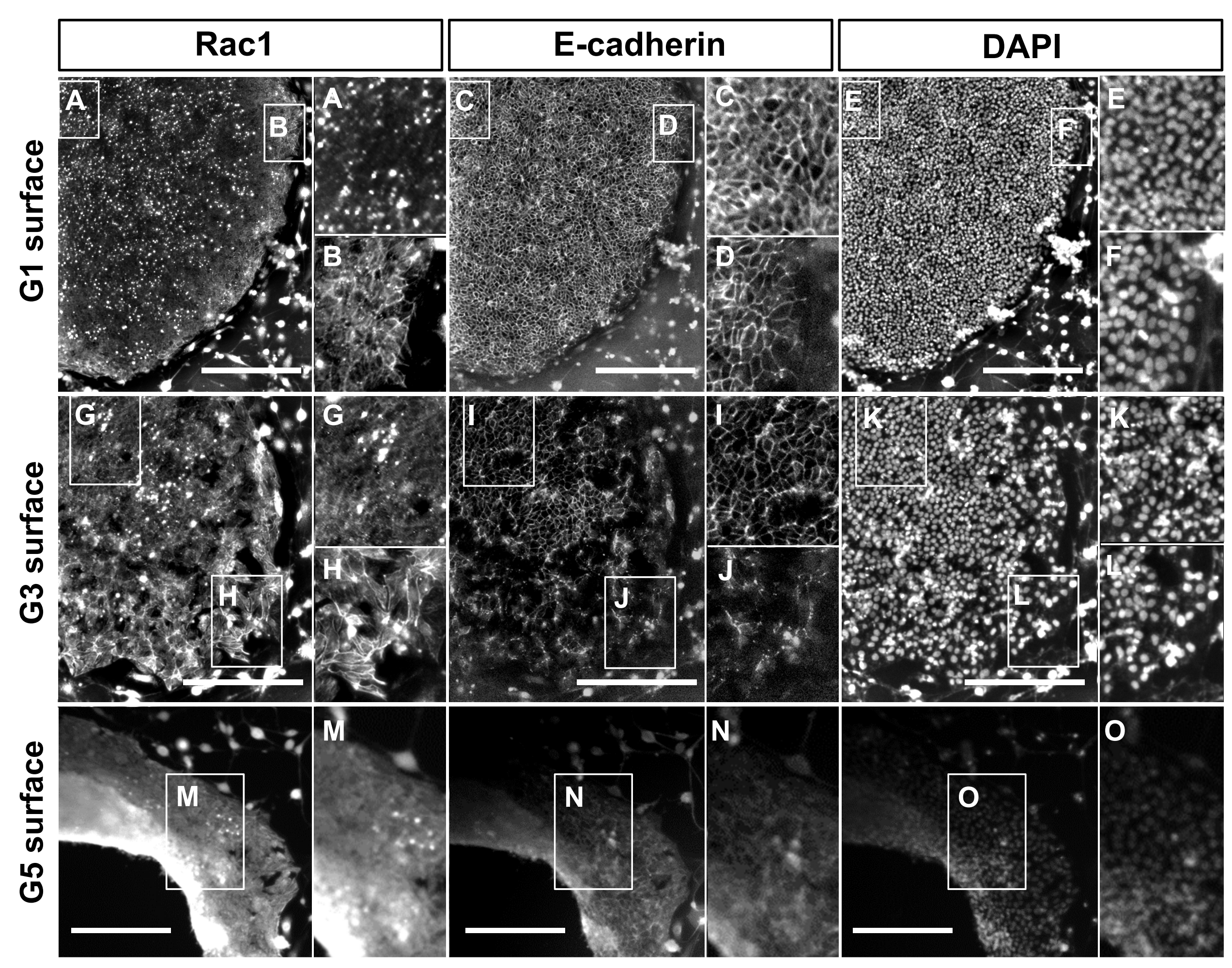




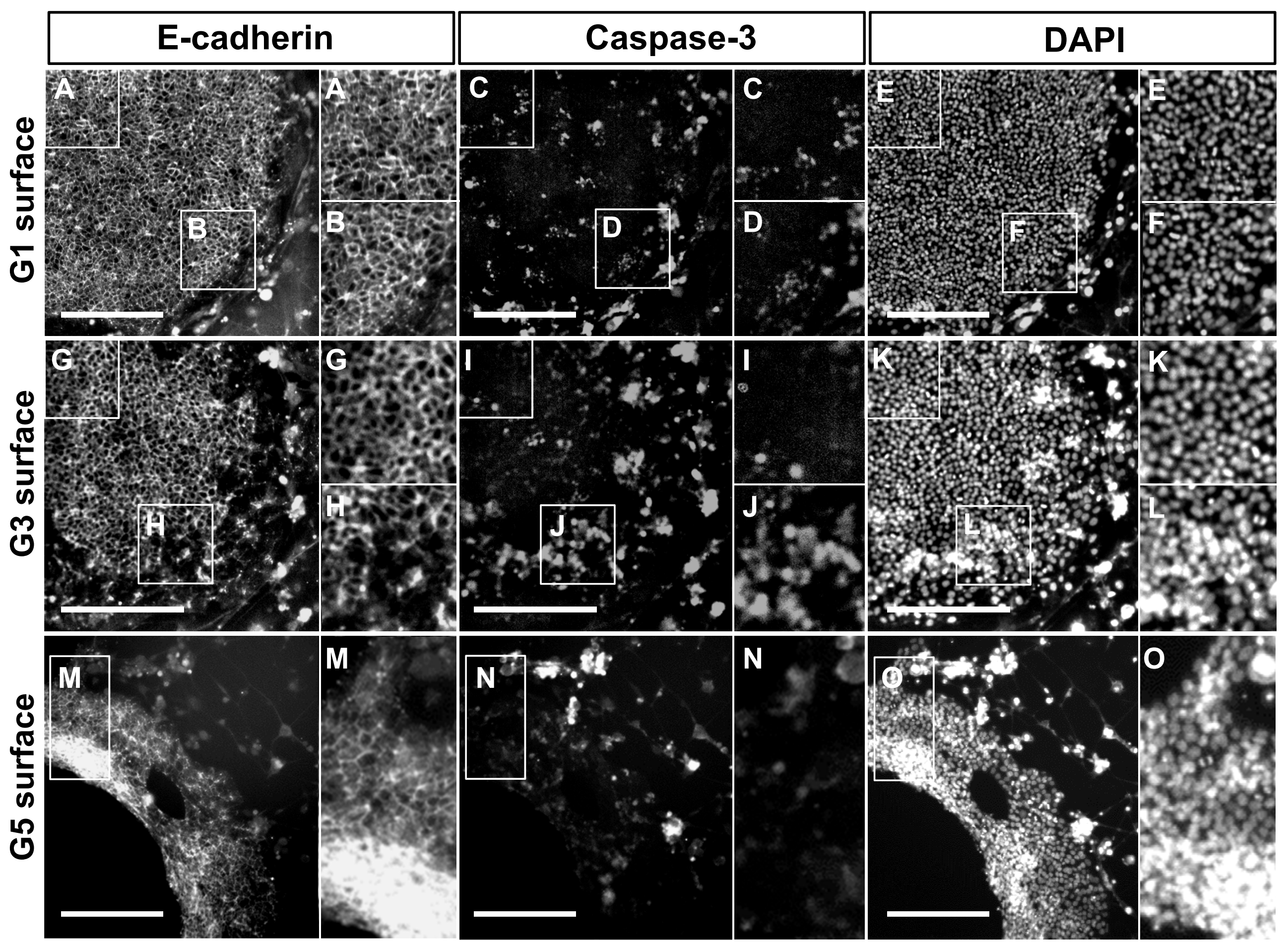


Figure 6

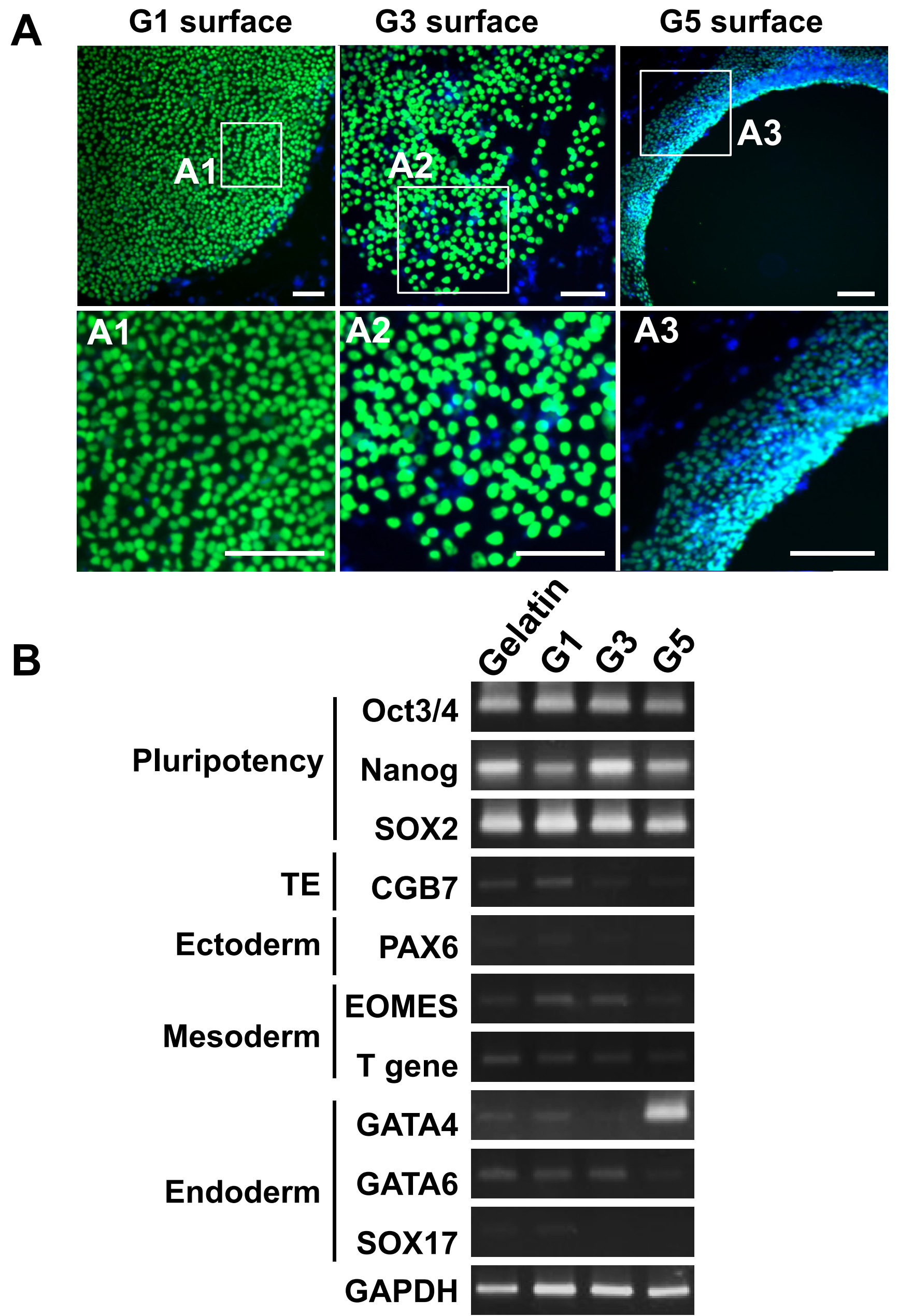




\section{Regulation of balances between cell-cell and cell-substrate interactions through altered migration}

\section{Switching hiPSC fate between self-renewal and early commitment lineages}

Cell-cell interactions Cell-substrate interactions

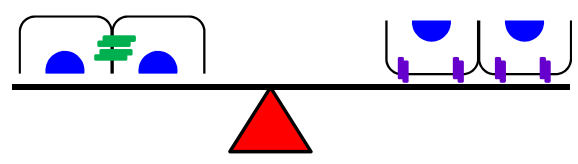

\section{Survival and undifferentiated state}

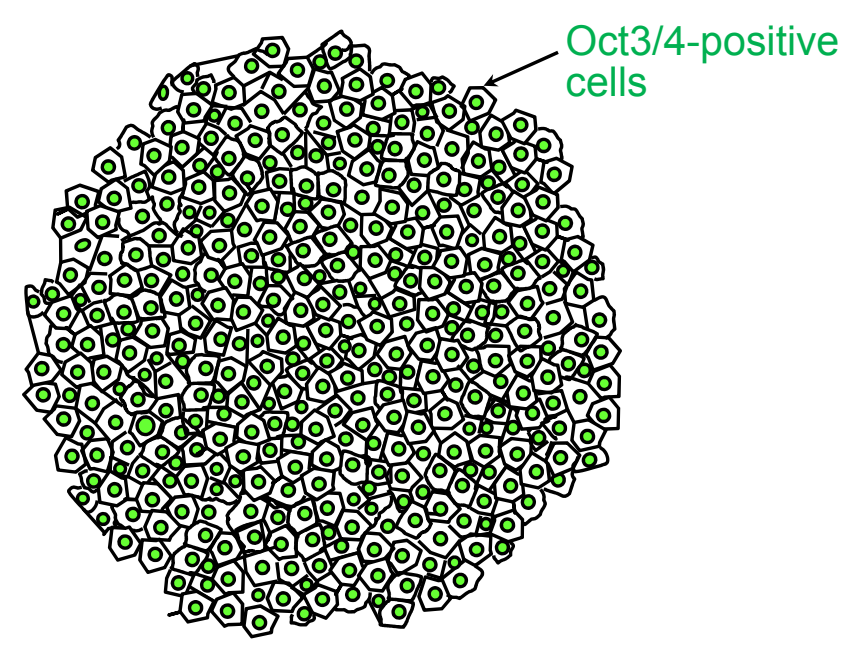

Maintenance of undifferentiated state and survival by keeping appreciate E-cadherin-mediated interaction
Caspase-3

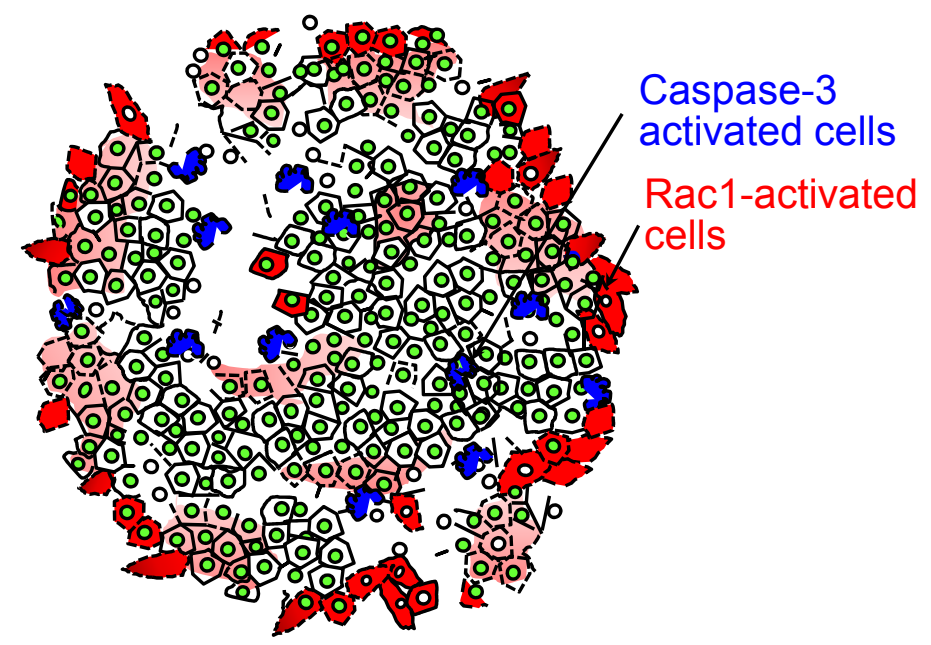

Generation of apoptotic cell death by loss of E-cadherin-mediated interactions
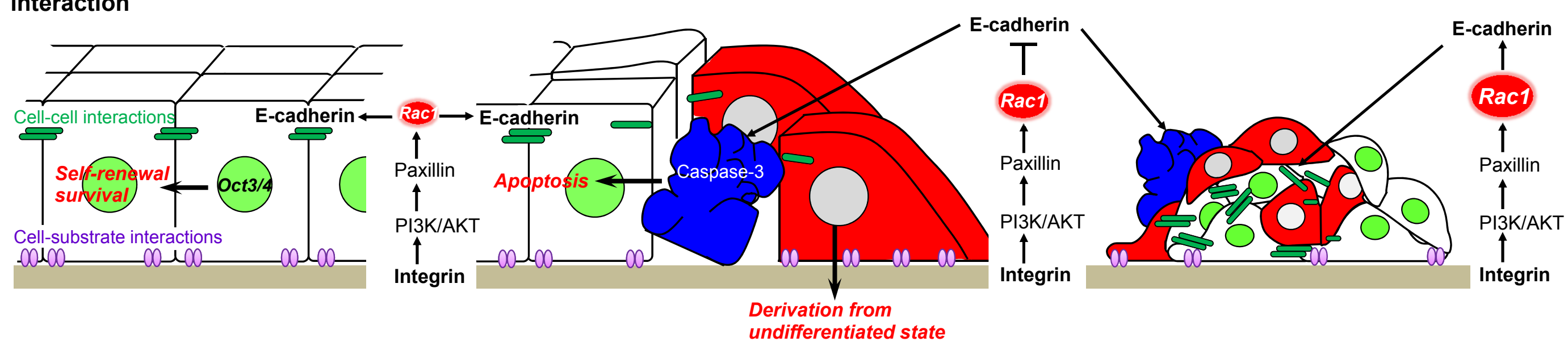

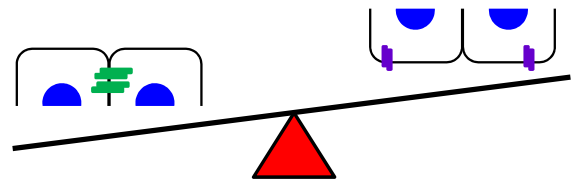

Early endoderm lineage

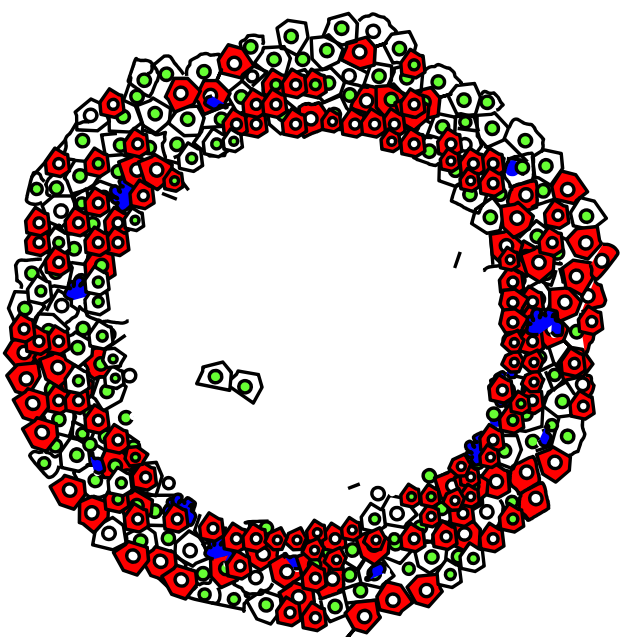

Generation of early endoderm lineage in aggregate formed by up-regulation of E-cadherin-mediated interactions 